\title{
Laser Nephelometric Equivalence Point Titration for the Measurements of Specific Proteins in Turbid Biological Fluids
}

\author{
By H. Hobler, U. Schütterle and S. Nickel \\ III. Medizinische Klinik und Poliklinik der Justus-Liebig-Universität Gießen, Gießen
}

(Received July 30/October 8, 1984)

Summary: A reliable, sentitive and specific laser nephelometric (LN) assay for the quantitative measurement of specific proteins in body fluids by equivalence point titration is described. The method is relatively free from interference encountered in conventional LN assays, e. g. unspecific background scatter, antigen excess, and the inhibition of complex formation by high ionic strength and salt concentration, low $\mathrm{pH}$ and elevated urea concentrations. It can be applied to turbid body fluids containing relatively small amounts of antigen. A serial dilution of the antigen preparation (human urinary albumin in the present work) is incubated with a fixed amount of highly diluted specific antiserum. The antigen concentration is determined by estimation of the equivalence point on the $\mathrm{LN}$ immunoprecipitation curve and comparison of this position with that on a standard curve. The data presented show good correlation with values obtained by radioimmunoassay $\left(\mathrm{p} \leqq 1 \times 10_{-} ; \log (\mathrm{LNET})=0.956 \log (\mathrm{RIA})-0.03 ; \mathrm{n}=50\right)$.

Lasernephelometrische Aquivalenzpunkt-Titration zur Messung spezifischer Proteine in trüben biologischen Flüssigkeiten

Zusammenfassung: Es wird ein zuverlässiges, sensitives lasernephelometrisches (LN) Verfahren für die quantitative und spezifische Bestimmung von Proteinen beschrieben. Von jeder Probe wird eine Verdünnungsreihe hergestellt, mit konstanter Menge eines spezifischen, hochverdünnten Antiserums inkubiert und über eine Bestimmung des Äquivalenzpunktes der LN-Immunpräzipitationskurve (Heidelberger-Kurve) die gesuchte Proteinkonzentration ermittelt. Bei diesem Verfahrensmodus sind die Fehlerquellen der herkömmlichen LNTechniken, wie unspezifische Hintergrundtrübung, Antigen-Exzeß, Hemmung der Antigen- und Antikörperkomplexbildung in den Proben durch hohe Ionenstärke und Salzkonzentration, niedriges $\mathrm{pH}$ sowie in Urinproben durch hohe Harnstoffkonzentration ohne wesentliche Bedeutung. Die Anwendbarkeit der Lasernephelometrie wird dadurch auf Proteinbestimmungen in Exkreten und Sekreten erweitert, in welchen wegen relativ kleiner Antigenkonzentration eine ausreichende Vorverdünnung der Proben zur Vermeidung von Störfaktoren nicht möglich ist. Das Verfahren wird am Beispiel der Bestimmung von Albumin im Urin erläutert. Ein Vergleich mit einem Radioimmunoassay für Urinalbumin zeigt eine gute Übereinstimmung $\left(p \leqq 1 \times 10_{-5}, \log (\mathrm{LNAT})=0,956 \log (\mathrm{RIA})-0,03 \cdot \mathrm{n}=50\right)$.

\section{Introduction}

In recent years, laser nephelometry $(\mathrm{LN})^{1}$ has increasingly won acceptance as a routine clinical diag-

\footnotetext{
1 Abbreviations:

Dilution (D), Laser nephelometry (LN), Laser nephelometric equivalence point titration (LNET).
}

nostic procedure. A wide spectrum of serum proteins can be quantitatively determined, including coagulation factors (1), complement factors (2) antiDNA antibodies (3) and the rheumatic factor (4). Various methods exist for the determination of immune complexes $(5,6)$, albumin and the $\gamma$-globulin fractions, $\operatorname{IgG}, \operatorname{IgA}$ and $\operatorname{IgM}(8,9)$. Since specific 
antisera are generally used, the specificity of $\mathrm{LN}$ assays is comparable to that of other immunological methods, e.g. radioimmunoassay and radial immunodiffusion. However, LN is simpler and more economical. Depending on the assay and turbidity of the sample, the lower limit of measurement is about $1 \mathrm{mg}$ antigen per liter native start material.

Since LN methods are based on the measurement of the intensity of scattered light, they are particularly suitable for clear liquids, e.g. cerebrospinal fluid (2, 9). $\mathrm{LN}$ is also applicable to serum, assuming that the concentration of desired antigen is large enough to allow dilution of the sample. The dilution of serum reduces unspecific background and the effect of various interfering factors, so that the conditions of measurement are similar to those for cerebrospinal fluid.

However, the conventional LN methods present problems when the concentration of the desired antigen is too small to allow prior dilution. In native material, various factors, e.g. unspecific background turbidity $(10,11)$, and in urine, the presence of urea (12), affect the formation of antigen-antibody complexes and therefore the intensity of scattered light. Measurements are therefore unreliable. Moreover, the usual LN methods can result in a false measurement of low antigen concentration when excess antigen is present. According to the Heidelberger curve (13), the formation of antigen-antibody complexes is reduced in the presence of excess antigen. In recognition of these problems, we developed the laser nephelometric equivalence point titration method (LNET).

In the following report, the LNET method is described, using as an example the determination of albumin in human urine. An increasing urinary excretion of albumin is often the first clinical sign of glomerular renal damage, e.g. in diabetes mellitus, where a latent diabetic nephropathy unmasked through physical stress may be detected by measurement of urinary albumin concentration (14). In this situation, albumin is a better early indicator of glomerular damage than $\beta_{2}$-microglobulins or light chain proteins (14). The normal range of urinary albumin excretion for healthy probands is about $10 \mathrm{mg} / 24$ hours $(17,18)$. Albumin concentrations in the order of $0.01 \mathrm{~g} / 1$ are too small to measure with the usual methods for detecting proteinuria, i.e. Albustix ${ }^{\oplus}$ or trichloroacetic acid precipitation. Until now, various radioimmunological methods have provided the only reliable procedures for the determination of urinary albumin in this low concentration $(15,16)$.

\section{Material and Methods}

\section{Apparatus}

We used a Hyland laser nephelometer (Hyland PDQ-TM Instrument, Travenol, Munich; Hyland Laboratories, Inc., Costa Mesa, Calif. 92626) with a helium-neon laser $(632.8 \mathrm{~nm})$. Long pipette tips, e.g. Bipette with $1 \mathrm{ml}$ Biotips (Schwarz-Mann, division of Becton: Dickinson \& Co., Orangenburg, New York, 10962, catalogue number 0010-30) are necessary to allow direct pipetting into the $\mathrm{LN}$ tubes.

\section{Reagents}

LN antisera, standard human serum for the standard dilution series and bovine albumin were obtained from the Behring Co. (Marburg), phosphate buffer $(0.005 \mathrm{~mol} / \mathrm{l}, \mathrm{pH} 7.4 ; 9.8$ mol $\left.\mathrm{KH}_{2} \mathrm{PO}_{4}+40.2 \mathrm{~mol} \mathrm{Na}_{2} \mathrm{HPO}_{4}\right)$ and $\mathrm{NaCl}$ solution $(0.15$ $\mathrm{mol} / \mathrm{l})$ containing bovine albumin $(1 \mathrm{~g} / \mathrm{l})$ were filtered through a Millipore $^{(\mathbb{D}}$ filter (Type HA $0.45 \mu \mathrm{m}$ )) before use. For comparison with LNET, 50 urine samples of patients with diabetes mellitus with radioimmunologically measured albumin concentrations were obtained through the courtesy of Prof. C. E. Mogensen, Department of Internal Medicine I, Amtssyghus, DK-8000 Århus C, Denmark.

\section{Conventional LN methods}

There are both kinetic and "end-point" LN methods. The kinetic methods measure the maximal rate of change of scattered light intensity after addition of the antibody to antigen. The period of incubation is a matter of seconds. This is advantageous for automated and continuous flow systems (17). In contrast, the "end-point" methods rely upon the achievement of an equilibrium state (incubation time: 30-60 minutes). In both methods, polyethyleneglycol is usually used as an adjuvant in order to accelerate the formation of antigen-antibody complexes. A standard dilution series is measured in the presence of excess antibody, the concentration of which is kept constant. Antigen concentration is determined by comparison of a measurement in an individual urine sample with the standard curve (for review, see l.c. (18)).

\section{LNET method}

LNET circumvents the effects of disturbing factors by using a modified manual "end-point" method. A dilution series for each urine sample is incubated with antibody in relatively low concentration Thus, for each urine, we not only make a single light scatter measurement in the presence of excess antibody, but also establish a $\mathrm{LN}$ immunoprecipitation curve over the entire range from antibody excess, through the equivalence point, to antigen excess. At the same time, a $\mathrm{LN}$ immunoprecipitation curve is established for a standard dilution series with a known concentration at equivalence point. Both sample and standard curves are measured under the same incubation conditions and with the same antibody concentration. Therefore, equivalence point represents the same antigen concentration in both curves. Since the antigen concentration at equivalence point is known for the standard curve, one must only take into consideration the dilution of the urine sample at equivalence point in order to determine the unknown protein concentration. Equivalence point is either interpolated or extrapolated.

\section{Sample preparation and LN measurement}

Phosphate buffer $(4.5 \mathrm{ml})$ is added to $0.5 \mathrm{ml}$ of native urine $\left(24 \mathrm{~h}\right.$ collection) and stored at $-20^{\circ} \mathrm{C}$ until analysis. After thawing, the sample is centrifuged at $10000 \mathrm{~g}$ for 10 minutes before LNET albumin determination. The procedure employed can be read from figure 1 . 


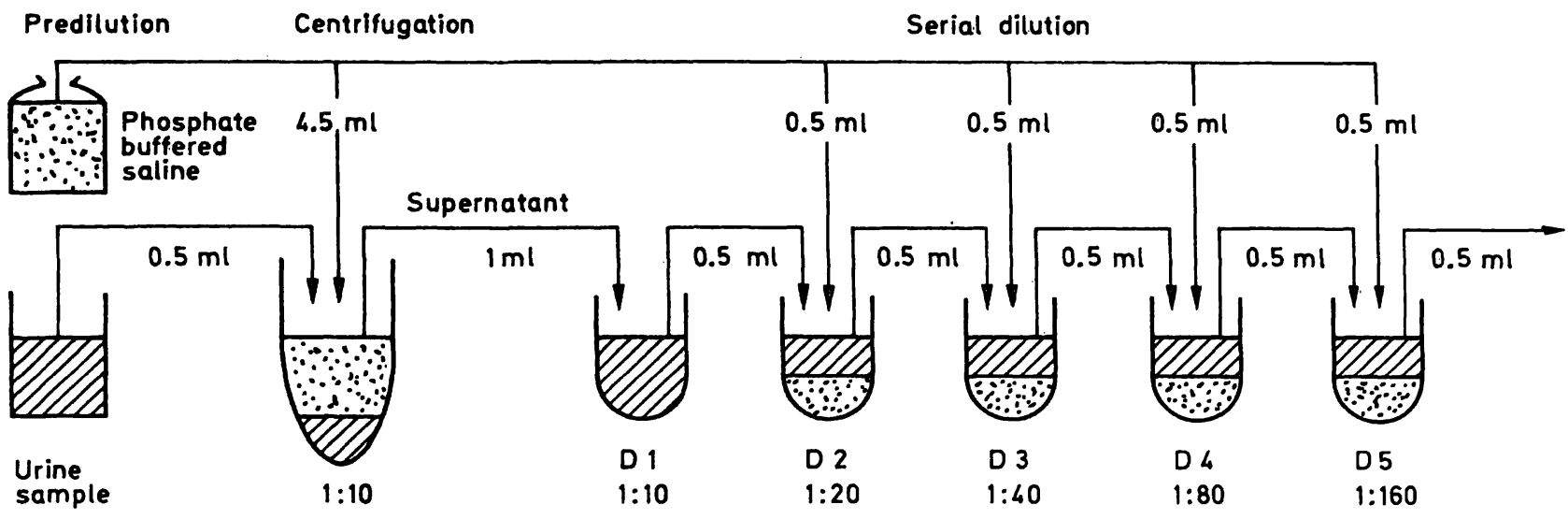

Fig. 1. Sample preparation.

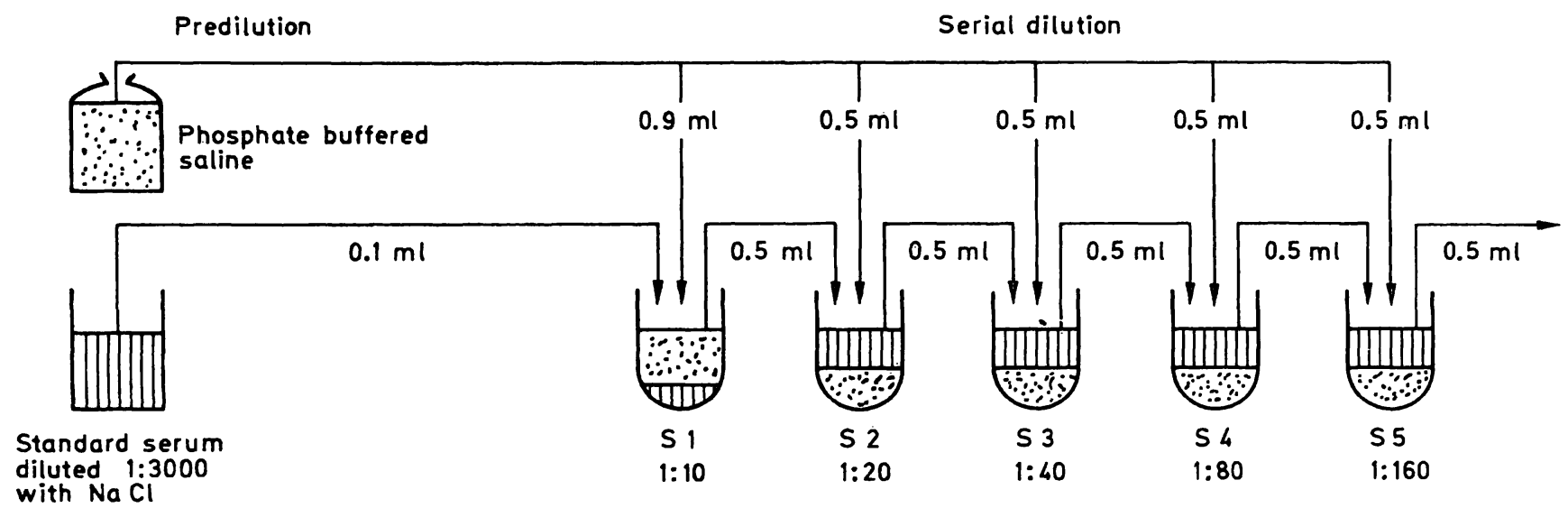

Fig. 2. Standard preparation

Briefly, a dilution series $\left(D_{1}-D_{5}\right)$ is prepared. Antigenantibody-reaction is started by addition of $0.5 \mathrm{ml}$ constant antiserum dilution. The antiserum dilution must be adapted to the required measuring range and usually lies between $1: 200$ and $1: 500$ (final dilution). Up to sixty minutes after the addition of antiserum, measurement of scatter light intensity gives the unspecific background turbidity, because in this period, formation of specific antigen-antibody complex is not detectable nephelometrically if the above mentioned relatively high antiserum dilution is used. After determination of unspecific background turbidity, the tubes are incubated for 12 hours at room temperature. Prior the final $\mathrm{LN}$ measurement, the samples are individually mixed with a rotary mixer for 1 to 2 seconds. After 2 to 3 minutes, the scatter light intensity is measured (sensitivity level III - IV).

\section{Calibration}

In order to determine which antigen concentration represents equivalence point, and to check if the antigen concentration of the sample lies within the range of direct measurement, a $L N$ immunoprecipitation curve is determined using a standard dilution series under the same incubation conditions (fig. 2).

The standard serum is diluted $1: 3000$ with $0.15 \mathrm{~mol} / 1 \mathrm{NaCl}$ to get an albumin concentration in the same range as that of normal urine.

Since the albumin concentrations of the standards are known, the dilution values can be plotted on a logarithmic scale (fig. 3).

The concentration of the antigen standard at equivalence point marks the lower border (D 1) of the field of direct measurement (interpolation of equivalence point) in the diagram (fig. 4).

\section{Determination of final result}

The standard curve (fig. 3 ) and the sample curves (fig. 4) are mirror images of one another, since the former is constructed from increasing concentrations plotted on a logarithmic antigen scale; whereas the sample curves are constructed from increasing dilutions (D 1 - D 5) plotted on a logarithmic scale. The sample dilutions (D 1-D 5) are plotted as seen in figure 4. Calibration is performed by placing D1 $(\mho)$ at the same antigen-concentration as the equivalence point $(\downarrow)$ of the standard curve (fig. 3). In analogy with the use of a slide rule (Wernier principle) the albumin concentration is determined, taking into acount the degree of dilution of the standard sample at equivalence point. For example, an equivalence point located exactly at D2 indicates that the concentration of albumin is double that of the standard antigen at equivalence point of the standard curve (fig. 3 and fig. 4).

Once a calibrated diagram is established, determination of the albumin concentration of the samples is very easy.

The intensities of scatter light of the 5 samples dilutions, D 1 trough D 5, are plotted in the standardized diagram (fig. 4) and the albumin concentration of the native urine can be directly read at equivalence point of the sample curve on the antigen scale without further calculation.

\section{Range of measurement}

Since LNET is a titration method performed in the presence of a constant antiserum concentration, the limits of measurement are dependent on antibody concentration. For instance if the antibody concentration is halved, the equivalence point of the standard curve ( $\sqrt{ }$ fig. 3 ) and $D 1 \sqrt{ }$, the limits of the window 


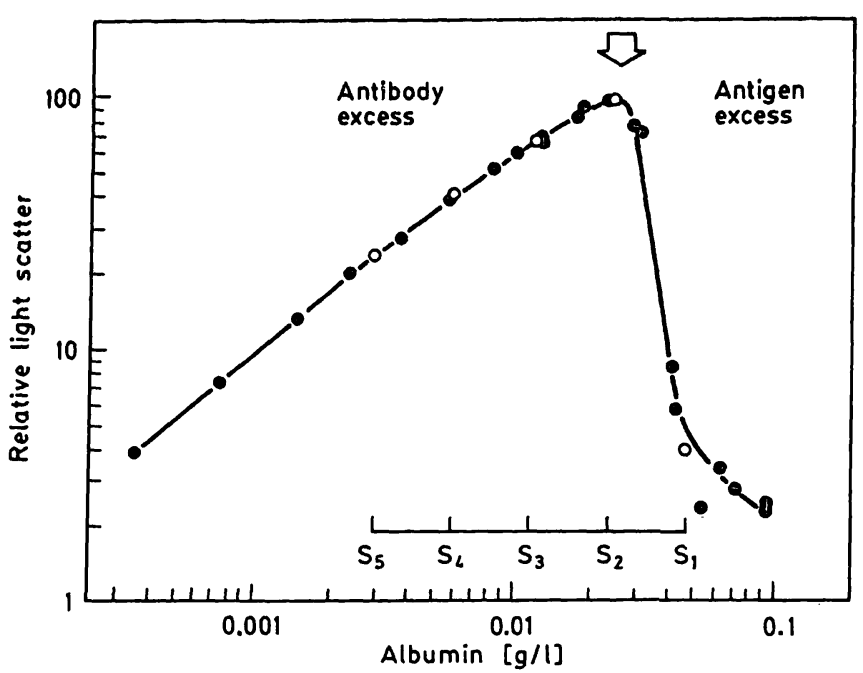

Fig.3. Typical standard curve of a LNET assay with serial dilution of antigen (standard serum albumin) and constant amount of antibody

(O) Routinely measured standard dilutions.

(๑) Additional standard dilutions for demonstration of the delineation at equivalence point. $(ß)$.

Reaction time $12 \mathrm{~h}$

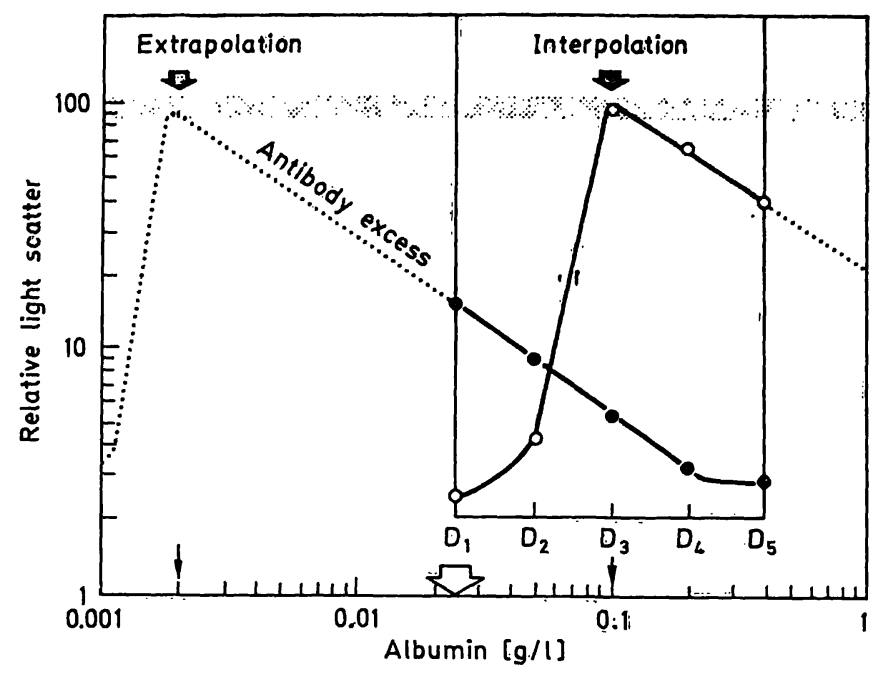

Fig. 4. Diagram for the determination of albumin concentration. The position of equivalence point (extrapolated) of sample $(0)$ indicates $0.002 \mathrm{~g} / \mathrm{l}$ albumin in native urine. The position of equivalence point (interpolated) of sample (O) indicates $0.1 \mathrm{~g} / 1$ albumin. The position of D $1(3)$ is determined by the position of equivalence point of the standard curve (fig. 3). Standard curve and sample curves are mirror images of one another (see text).

antibody excess (without adjuvant!) and stable equivalence point localization. Unspecific aggregation during the long incubation is not a significant problem in view of the relatively large antiserum dilution. Sedimentation effects are negligible if the samples are mixed with a rotary mixer before the LN measurement.

\section{Adjuvants}

The usual LN methods are often performed in the presence of adjuvants. Protamine $(19,20)$, and polymers like dextran (21) and polyethyleneglycol (22) accelerate the formation of antigenantibody complexes, leading to a linear LN immunoprecipitation curve within 1 hour. All these adjuvants result in displacement of equivalence point into the higher antigen range. Moreover, dextran and polyethyleneglycol also flatten the $\mathrm{LN}$ immunoprecipitation curve, making it difficult to assign equivalence point exactly to a given antigen concentration and thus hampering the extrapolation of the desired antigen concentration. In contrast, protamine does not have this disadvantage, but its effect is strongly dependent on the ionic strength of the incubation environment. Since individual urine samples vary widely in ionic concentration, comparison of the sample with the standard is questionable when protamine is used.

In view of the above mentioned disadvantages, we chose to omit the use of adjuvants in the LNET albumin assay.

\section{Results}

\section{Accuracy of measurement}

The end results are determined graphically. After LN measurement, the scattered light intensities of the sample dilution series are plotted on a standardized diagram. After extrapolation of equivalence point, the desired antigen concentration in read directly at 
the level of equivalence point on the appropriate antigen scale (albumin concentration in the present example). The degree of dilution of the urine sample at equivalence point is determined by a diagram as described (fig. 4). The use of a logarithmic scale improves the accuracy of reading. Two trends work in opposite directions with respect to their advantages and disadvantages. Low native antigen concentrations limit the extent to which the sample can be diluted. The significance of interfering factors, e.g. unspecific background turbidity, etc., is therefore greater. This disadvantage is compensated for by the logarithmic expansion of the antigen scale in the low antigen range, which improves the accuracy of reading. High native antigen concentrations allow dilution of the sample, which reduces the effect of perturbants at equivalence point. However, this advantage is counteracted by the poor readability caused by the logarithmic narrowing of the antigen scale in the high antigen range. As a consequence of these opposing trends, the conditions of measurement are best in the middle and low concentration ranges.

\section{Precision}

The precision of the LNET method was tested in 10 urine samples analysed in quintuplicate during a single run. In 5 of these samples the albumin concentrations lay between 0.003 and $0.009 \mathrm{~g} / 1$, and the equivalence point was extrapolated. In the other 5 samples, the albumin concentrations lay between 0.01 and $0.07 \mathrm{~g} / \mathrm{l}$, and the equivalence point was interpolated. The resulting arithmetic means and standard deviations were found (tab. 1).

The interassay variance was $<5 \%$. It should be noted that the antibody concentrations vary considerably between different commercial lots! For each assay a new calibration has to be performed.

\section{Recovery}

In recovery experiments, lyophilised human albumin was added to 20 urine specimens to make a final albumin concentration of $0.005 \mathrm{~g} / 1$ native urine. The starting albumin concentrations were extrapolated in
Tab. 1. Precision of the laser nephelometric equivalence point titration. Ten urine samples were analysed in quintuplicate during a single run.

\begin{tabular}{|c|c|c|c|}
\hline \multirow[t]{2}{*}{ No. } & \multicolumn{2}{|c|}{$\begin{array}{l}\text { Albumin } \\
\overline{\mathbf{x}}\end{array}$} & \multirow[t]{2}{*}{$\mathrm{CV}$} \\
\hline & $(\mathrm{mg} / \mathrm{l})$ & $(\mathrm{mg} / \mathrm{l})$ & \\
\hline \multicolumn{4}{|c|}{ Extrapolated values } \\
\hline 1 & 9.0 & 0.14 & 0.02 \\
\hline 2 & 1.0 & - & - \\
\hline 3 & 4.3 & 0.21 & 0.05 \\
\hline 4 & 4.6 & 0.16 & 0.03 \\
\hline 5 & 3.5 & 0.08 & 0.02 \\
\hline \multicolumn{4}{|c|}{ Interpolated values } \\
\hline 1 & 29.6 & 0.36 & 0.01 \\
\hline 2 & 68.0 & 10.88 & 0.16 \\
\hline 3 & 42.0 & 9.24 & 0.22 \\
\hline 4 & 11.6 & 1.58 & 0.14 \\
\hline 5 & 70.6 & 0.73 & 0.01 \\
\hline
\end{tabular}

$\overline{\mathrm{x}}=$ arithmetic mean

$\mathrm{s}=$ standard deviation

$C V=$ coefficient of variation, $\frac{s}{\bar{x}}$

10 samples and interpolated in 10 samples. After equilibration, the measured and expected albumin concentrations were compared. Theoretically, no significant difference should exist between the observerd and expected values. This hypothesis $\left(\mathrm{H}_{0}\right)$ was tested with the t-test for dependent samples. No difference could be demonstrated for extrapolated values. The calculated probability of error was $p=0.26$ for extrapolated values, and $p=0.05$ for interpolated values.

\section{Reliability}

The reliability was tested by comparing values determined by LNET with those determined by radioimmunoassay (RIA) (15). The agreement between the extrapolated and interpolated values determined by LNET and those measured by RIA was tested using regression analysis. All values were expressed logarithmically to improve the fit to the normal distribution. The linear regression and probability of error were calculated separately for the group of extrapolated values $(\min .=1.1 \mathrm{mg} / \mathrm{l} ; \max .=7.0$;

Tab. 2. Reliability of the laser nephelometric equivalence point titration (LNET). Values determined by LNET were compared . with those obtained by RIA.

\begin{tabular}{llll}
\hline Group & $\mathrm{n}$ & Regression line & $\begin{array}{l}\text { Probability } \\
\text { of Error }\end{array}$ \\
\hline Extrapolated values & & & $\mathrm{p}=0.00005$ \\
Interpolated values & 27 & $\log (\mathrm{LNET})=0.911 \log (\mathrm{RIA})+0.06$ & $\mathrm{p}=0.00005$ \\
All values & 23 & $\log (\mathrm{LNET})=0.866 \log (\mathrm{RIA})+0.02$ & $\mathrm{p}=0.00005$ \\
\hline
\end{tabular}


$\left.X_{\text {med. }}=3.3 \mathrm{mg} / \mathrm{l}\right)$ and for the group of interpolated values $\left(\min .=8.0 \mathrm{mg} / \mathrm{l} ; \max =105 \mathrm{mg} / \mathrm{l} ; X_{\text {med. }}=23\right.$ $\mathrm{mg} / \mathrm{l} ; \mathrm{n}=23$ ). It was then tested if a common linear regression could be assumed for both groups. The results are summarized in table 2.

Moreover, it was checked if the calculated regression coefficient approximated to unity, i.e. the agreement between measurement should be independent of the point studied. Significant deviation from unity whould indicate an inherent error in the LNET method (tab. 3).

Tab. 3. Regression line characteristics (cf. tab.2).

Confidence interval

Regression coefficient $(0.896,1.011)$

Intercept on the $y$-axis

Since the expected regression coefficient (1) and the expected intercept $(0)$ are contained within the interval of confidence, it may be assumed that the calculated regression line approximates to that expected: log $(\mathrm{LNET})=1 \times \log (\mathrm{RIA})+0$; and that $\mathrm{LNET}=$ RIA.

\section{Discussion}

\section{The role of interferences}

LNET eliminates most of the usual interferences common to conventional LN methods. A major problem of the latter is presented by the poor transmittance of the beam through a sample, due to increased background. This is particularly serious when dilution of the specimen is not possible, due to low antigen concentration. Circulating immune complexes unrelated to the antigen-antibody system under investigation also constitute a source of error in conventional LN methods (23). The commonly practiced subtraction of the background scatter light intensity from that due to formation of specific immune complexes is only an approximate correction, since the intensity of scattered light is not strictly additive.

Ions exert a strong influence on the formation of antigen-antibody complexes and constitute a serious source of error if differences in ionic strength and salt concentration exist between standard and sample. Increasing ionic strenght inhibits complex formation in that the reaction velocity and final size of the immune complexes are reduced by decreased electrostatic attraction between the reactants (10). This

inhibitory effect is dependent on the salt concentration, anion size and radius of hydration (tab. 4).

Tab. 4. Influence of ions on the formation of antigen-antibody complexes.

\begin{tabular}{c}
\hline $\mathrm{F}^{-}<\mathrm{Cl}^{-}<\mathrm{Br}^{-}<\mathrm{NO}_{3}^{-}$ \\
$\mathrm{Na}^{+}$salt $<\mathrm{K}^{+}$salt \\
$0.1 \mathrm{~mol} / 1<0.5 \mathrm{~mol} / 1$
\end{tabular}

Assuming a common anion, potassium șalts are more inhibitory than sodium salts. $\mathrm{NaF}$ is an exception in that it augments the formation of antigen-antibody complexes more in higher than in lower concentrations (12). Differences in $\mathrm{pH}$ have relatively little effect on the formation of antigen-antibody complexes. A negative effect on complex formation cannot be expected, unless $\mathrm{pH}$ values lie outside the range $\mathrm{pH}$ $6-8$ (10). Such interference is generally avoidable by use of buffers (see fig. 1).

The above mentioned interfering factors affect the course of the LN immunoprecipitation curve by either increasing or decreasing the intensity of scatter light as a whole (24). However, they do not influence the position of equivalence point on the antigen scale and consequently have no effect on LNET, when the equivalence point of the sample dilution curve is interpolated. For the extrapolated results the influence of interference is mostly negligible because of the logarithmic expansion of the antigen scale. Only in individual cases is a subtraction of the background turbidity unavoidable. In the described urine albumin assay no subtraction was performed.

Organic polycations and polymers can accelerate specific antigen-antibody reactions and displace equivalence point into the high antigen range. Both effects are demonstrable by laser nephelometry for protamine chloride in concentration of $0.005 \mathrm{~g} / 1$ (19). Polycationic effects are strongly dependent on the ionic strength of the incubation medium. In contrast, the analogous effects of polyethyleneglycol (22) and dextran (21), which are explained by steric exclusion $(25,26)$, are independent of $\mathrm{pH}$, ionic strength and absolute salt concentration. From a methodological standpoint, these phenomena are only interesting if the substances are used as adjuvants.

The inhibitory effect of urea and its significance as a possible interfering substance have been recognized for a long time (12). Our findings demonstrate that high concentration of urea can also displace the equivalence point. A urea concentration of $1.6 \mathrm{~mol} / \mathrm{1}$ which can be found in the urine during catabolic 


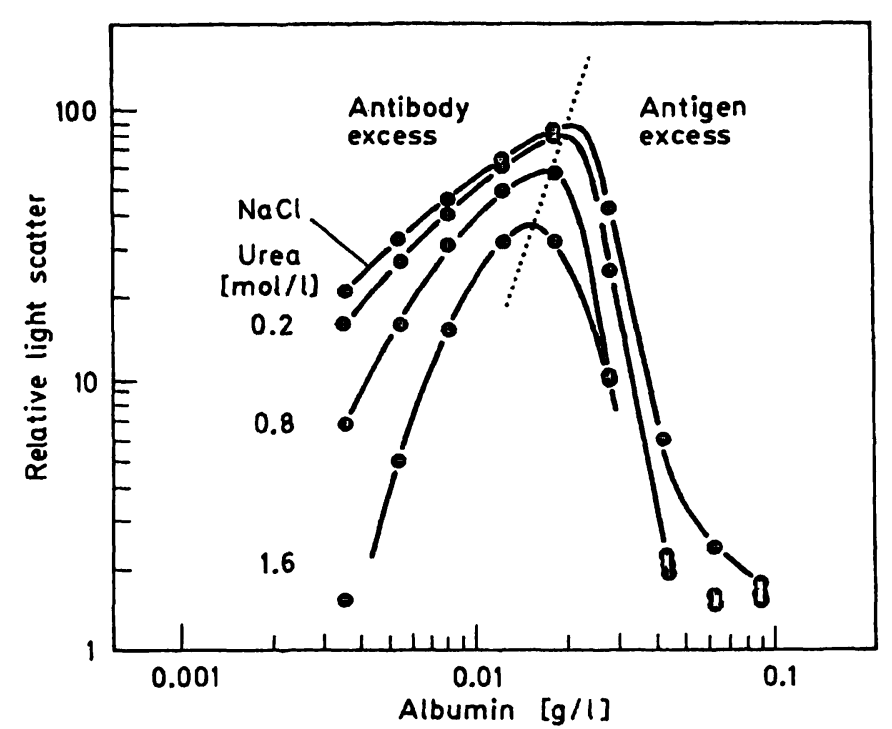

Fig. 5. Laser nephelometric equivalence point titration of standard serum- $\mathrm{NaCl}$ albumin. High concentrations of urea $(0.2-1.6 \mathrm{~mol} / \mathrm{l})$ reduce complex formation and displace the equivalence point to low antigen range. Reaction time $60 \mathrm{~min}$.

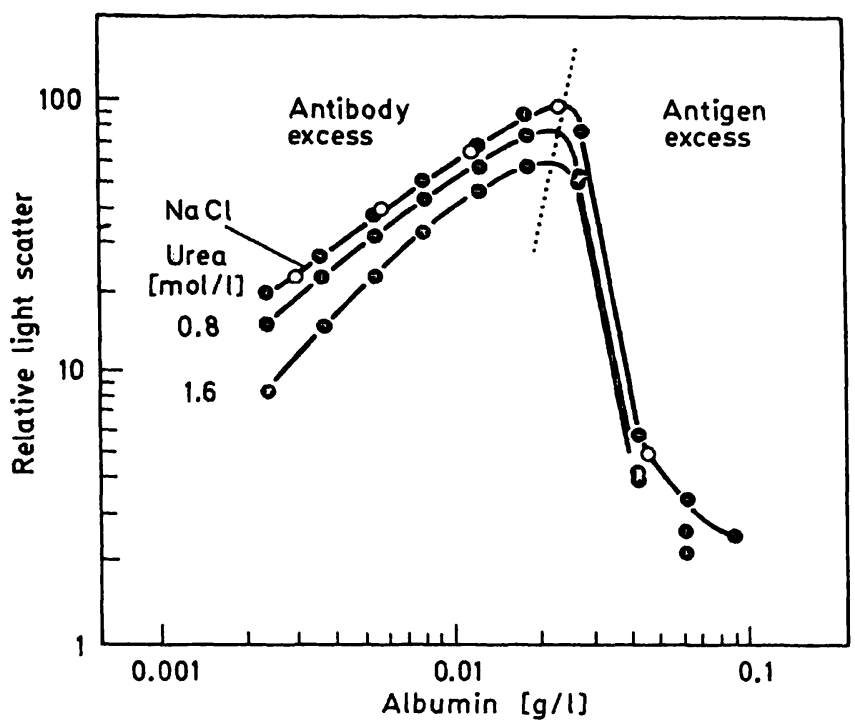

Fig. 6. Laser nephelometric equivalence point titration of standard serum- $\mathrm{NaCl}$ albumin. Comparison with figure 5 shows that the inhibitory effect of urea $(0.2-1.6 \mathrm{~mol} /$ l) is less evident with longer incubation time $(12 \mathrm{~h})$.

excess in the field of measurement is easily recognized and thus constitutes no significant source of error.

\section{Assignement of equivalence point}

One of the prerequisites for LNET is an optimal system which allows exact assignment of equivalence point to a given antigen concentration. In the literature, the description of the immunoprecipitation curve is generally confined to an equivalence region $(11,13,21,22)$ rather than to an equivalence point. Small incremental changes in antigen concentration demonstrate that the relatively large dilution of the antiserum used causes the LN immunoprecipitation curve to "hook", rather than curve at the equivalence point (fig. 3). The relatively long incubation time assures linearity of the $\mathrm{LN}$ immunoprecipitation curve in the antibody-excess range. Both effects facilitate the exact localization of equivalence point on the antigen scale.

In the range of antibody-excess, the antigen-antibody complexes are stable, allowing reproducible $L N$ measurement. In a large number of antigen-antibody systems studied, the slopes of the LN immunoprecipitation curves in the range of antibodyexcess, given a sufficiently long incubation time, were practically the same. The complexes are much less stable, however, in the antigen-excess range. The slopes of the LN immunoprecipitation curves in the range of antigen-excess showed great variability depending on the antiserum used. The finding that the variation of slope is clearly greater in the range 


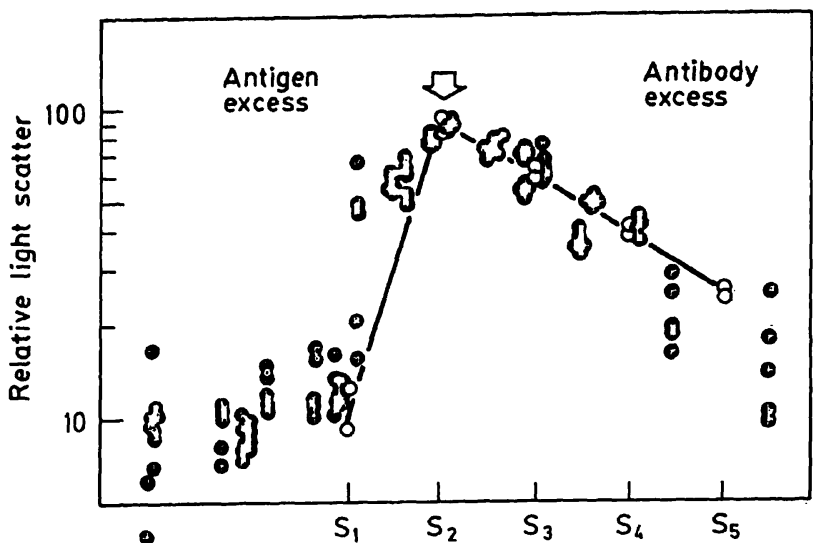

Fig. 7. Values from $5 \mathrm{LN}$ immunoprecipitation curves (O) performed in a single experimental run superimposed on the LNET standard curve $(O)$. Quintuplicate determinations were performed on each of the 5 dilutions of 5 urine samples. The individual variation and deviation of measured values from the standard curve are greatest in the antigen-excess range, and smallest at the equivalence point $(\Omega)$

of antigen-excess than in that of antibody-excess (fig. 7) implies that the relative light scattering values in the antibody-excess range are more valuable for the determination of equivalence point in LNET.

\section{Comparison with other methods}

Compared with conventional LN methods, LNET is performed with a relatively highly diluted antiserum. The described LNET assay, using an antiserum final dilution of $1: 500$, requires only $10 \mu$ antiserum for the albumin determination in one urine sample. LNET is a relatively economical procedure.

As a manual procedure, LNET is time-consuming, since a dilution series $(n=5)$ must be established for each sample. A simplified microplate system or automation would be desirable technical improvements. One can compare LNET with a quintuplicate determination of a given sample using conventional LN procedures. However, LNET has the advantage that measurements are performed with various antigen-antibody ratios. Since a complete curve is always determined, each of the 5 measurements can be judged in context of the other values. Pipetting errors, measuring mistakes and the effects of interference are therefore easily recognizable.

LNET with a 12 hour incubation period and the kinetic $\mathrm{LN}$ with an incubation period of seconds (17) constitute the polar extremes found among laser nephelometric procedures. One has to consider that the kinetics of the antigen-antibody reaction are strongly influenced by inhibitors, as well as by the use of adjuvants. With long incubation periods, the

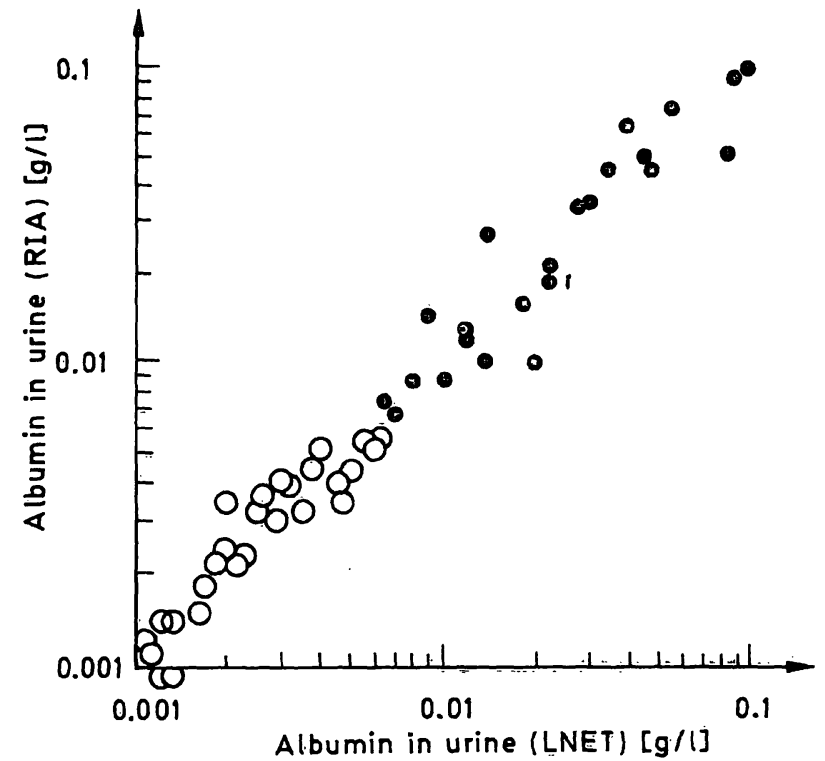

Fig. 8. Comparison of albumin concentrations determined by radioimmunoassay (RIA) (17) and laser nephèlometric equivalence point titration method (LNET) of 50 urine specimens from diabetic patients.

(O) extrapolated $(n=27),(0)$ interpolated $(n=23)$. No subtraction of background turbidity was performed. $\left(p=1 \times 10_{-5} ; \log (\right.$ LNET $)=0.956 \log ($ RIA $)-0.03$; $\mathrm{n}=50)$.

effect of inhibitors upon the degree of lattice formation is decreased. Whether such interfering factors are relevant to kinetic assays under extreme measurement condition remains to be statistically evaluated for the individual systems. With LNET, however, the presence of interfering factors can be immediately detected by the shape of the individual sample immunoprecipitation curve.

A comparison of LNET with radioimmunoassay (17) shows good agreement (fig. 8).

\section{Application}

LNET is applicable to the nephelometric analysis of proteins in turbid body fluids, supernatants of cell cultures etc.

A precipitating antiserum of high avidity (capable of lattice formation) is essential for an assay. Ritchie (29) noticed that antisera, which appeared to be of excellent quality for in-gel-techniques, were sometimes absolutely functionless in nephelometric techniques. This makes LNET suitable for differentiating, in fluid phase, between lattice forming antisera and others without this capacity. Monoclonal antibodies are suitable for LNET, only if they are used as mixtures of at least two different monoclonal antibody popu= lations without mutual sterical hindrance (30).

We always recommend LNET when there are doubts about the reliability of conventional LN methods. 
The superposition of various sample curves in figure 7 demonstrates the lack of agreement due to errors of pipetting, measurement, and differences in incubation media. The deviation from the standard curve is greater, the further one gets from equivalence point. It is greatest in the range of antigen-excess where soluble antigen-antibody complexes are found, suggesting that interfering factors in the incubation media play an important role. The logarithmic scale gives good agreement at equivalence point (fig. 7). It is therefore logical to prefer equivalence point itself for the determination of antigen or antibody, rather than a single turbidity reading in the range of antibodyexcess. The simpler LN methods are, of course, preferable, if the immunoprecipitation curves of individual samples are uniform and congruent with the standard curve. Our investigations showed that this was not the case for the determination of albumin in urine with conventional techniques and we now use LNET only. LNET represents an adjunct to the conventional $\mathrm{LN}$ methods and expands the field of usefulness of laser nephelometry.

\section{References}

1. Schmith-Hueber, U., Nachbar, J. \& Asbeck, F. (1980) J. Clin. Chem. Clin. Biochem. 18, $221-225$.

2. Sieber, A. (1977) Laborpraxis Laboratoriumsblätter Behringwerke AG 27, 109-112.

3. Lakomek, H. J., Jacobi, E., Husmann, K. \& Krüskemper H. L. (1979) Dtsch. Med. Wochenschr. 104, 980-982.

4. Jones, C. E., Rousseau, R. J. \& Maxwell (1979) Am. J. Clin. Pathol. 72, 432-436.

5. Höffken, K., Bestek, U., Sperberg, U. \& Schmidt C. G. (1979) J. Immunol. Meth. 29, 237-244.

6. Krapf, F., Renger, D., Schedel, I., Leiendecker, K., Leyssens, H. \& Deicher, H. (1982) J. Immunol. Meth. 54, 107-117.

7. Ritchie, R. F. (1975) Fed. Proc. 34, 2139-2144.

8. Schultze, H. E. \& Schwick, G. (1959) Clin. Chim. Acta 4, $15-25$.

9. Killingsworth, L. M. \& Savory, J. (1973) Clin. Chim. Acta $43,229-282$

10. Marrack, J. R. \& Richards, C. B. (1971) Immunology 20, 1019-1039.

11. Tengerdy, R. P. (1967) J. Immunol. 99, 126-132.

12. Killingsworth, L. M. \& Savory, J. (1973) Clin. Chem. 19, 403-407.

13. Heidelberger, M. \& Kendall, F. E. (1935) J. Exp. Med. 62, 697-719.

14. Mogensen, C. E., Vittinghus, E. \& Solling, K. (1969) Kidney Internat. 16, 385-393.

15. Miles, D. W., Mogensen, C. E. \& Gundersen, H. J. G. (1970) Scand. J. Clin. Lab. Invest. 26, 5-11.

16. Woo, J., Floyd, M., Cannon, D. C. \& Kahan, B. (1978) Clin. Chem. 24, 1464-1467.

17. Sternberg, J. C. (1977) Clin. Chem. 23, 1456-1464.

18. Deverill, I. \& Reeves W. G. (1980) J. Immunol. Meth. 38 , $191-204$.

19. Hobler, H. (1984) Immunität und Infektion 12, 111-112.

20. Hobler, H. \& Pflughaupt, K. W. (1979) J. Clin. Chem. Clin. Biochem. 17, 508.

21. Hellsing, K. (1969) Biochem. J. 112, 475-481.

22. Lizana, J. \& Hellsing, K. (1974) Clin. Chem. 20,1181-1186.

23. Ziegler, G. B. \& Strobel, G. J. (1978) Diagnostik und Intensivtherapie 3, 74-76.

24. Pflughaupt, K. W., Hobler, H. \& Schmitt, J. (1979) Fortschr. d. techn. Medizin in der Neurolog. Diagnostik und Therapie, Wien.

25. Ogston, A. G. (1958) Trans. Faraday Soc. 54, 1754-1757.

26. Ogston, A. G. \& Phelps, C. F. (1961) Biochem. J. 78, $827-833$.

27. Anderson, R. J. \& Sternberg, J. C. (1978) In: Automated immunoanalysis, Part 2, (Ritchie, R. F. ed.) Marcel Dekker Inc., New York, p. 409.

28. Deverill, J. (1979) Protides Biol. Fluids 26, 697-700.

29. Ritchie, R. F. (1973) Protides. Biol. Fluids 21, 569-578.

30. Jeffris, R., Deverill, J., Ling, N. R. \& Reeves, W. G. (1980) J. Immunol. Meth. 29, 227-230.

31. Hobler, H., Keller, M. \& Weise, M. (1980) Nieren- und Hochdruckkrankheiten, Nr. 5, p. 209.

Dr. H. Hobler

III. Med. Klinik und Poliklinik

Justus-Liebig-Universität Gießen

Rodthohl 6

D-6300 Gießen 
